OPEN ACCESS

Edited by:

Anne Hilgendorff,

Ludwig Maximilian University of

Munich, Germany

Reviewed by:

Jean-Paul Janssens,

Geneva University Hospitals

(HUG), Switzerland

Stéphane Doutreleau,

Centre Hospitalier Universitaire de

Grenoble, France

*Correspondence:

Michael Furian

michael.furian@usz.ch

Specialty section: This article was submitted to

Pulmonary Medicine,

a section of the journa

Frontiers in Medicine

Received: 30 April 2020 Accepted: 25 January 2021

Published: 01 March 2021

Citation:

Bisang $M$, Latshang $T D$, Aeschbacher SS, Huber F, Flueck D, Lichtblau M, Ulrich S, Hasler ED,

Scheiwiller PM, Ulrich S, Bloch KE and Furian M (2021) Nocturnal Heart

Rate and Cardiac Repolarization in Lowlanders With Chronic Obstructive Pulmonary Disease at High Altitude:

Data From a Randomized

Placebo-Controlled Trial of Nocturnal Oxygen Therapy.

Front. Med. 8:557369.

doi: 10.3389/fmed.2021.557369

\section{Nocturnal Heart Rate and Cardiac Repolarization in Lowlanders With Chronic Obstructive Pulmonary Disease at High Altitude: Data From a Randomized, Placebo-Controlled Trial of Nocturnal Oxygen Therapy}

\author{
Maya Bisang, Tsogyal D. Latshang, Sayaka S. Aeschbacher, Fabienne Huber, \\ Deborah Flueck, Mona Lichtblau, Stefanie Ulrich, Elisabeth D. Hasler, \\ Philipp M. Scheiwiller, Silvia Ulrich, Konrad E. Bloch and Michael Furian* \\ Department of Respiratory Medicine, Sleep Disorders Center, University Hospital of Zurich, Zurich, Switzerland
}

Background: Chronic obstructive pulmonary disease (COPD) is associated with cardiovascular disease. We investigated whether sleeping at altitude increases nocturnal heart rate $(\mathrm{HR})$ and other markers of cardiovascular risk or arrhythmias in lowlanders with COPD and whether this can be prevented by nocturnal oxygen therapy (NOT).

Methods: Twenty-four COPD patients, with median age of 66 years and forced expiratory volume in $1 \mathrm{~s}\left(\mathrm{FEV}_{1}\right) 55 \%$ predicted, living <800 m underwent sleep studies at Zurich $(490 \mathrm{~m})$ and during 2 sojourns of 2 days each at St. Moritz $(2,048 \mathrm{~m})$ separated by 2 -week washout at $<800 \mathrm{~m}$. During nights at $2,048 \mathrm{~m}$, patients received either NOT (2,048 $\mathrm{m} \mathrm{NOT)} \mathrm{or} \mathrm{ambient} \mathrm{air} \mathrm{(2,048} \mathrm{m} \mathrm{placebo)} 3 \mathrm{~L} / \mathrm{min}$ via nasal cannula according to a randomized, placebo-controlled crossover trial. Sleep studies comprised ECG and pulse oximetry to measure HR, rhythm, HR-adjusted QT interval (QTc), and mean oxygen saturation $\left(\mathrm{SpO}_{2}\right)$.

Results: In the first nights at $490 \mathrm{~m}, 2,048 \mathrm{~m}$ placebo, and 2,048 m NOT, medians (quartiles) of $\mathrm{SpO}_{2}$ were $92 \%(90 ; 94), 86 \%(83 ; 89)$, and $97 \%(95 ; 98)$ and of HR were 73 (66; 82), 82 (71; 85), and 78 bpm (67; 74) ( $P<0.05$ all respective comparisons). QTc increased from $417 \mathrm{~ms}(404 ; 439)$ at $490 \mathrm{~m}$ to $426 \mathrm{~ms}(405 ; 440)$ at $2,048 \mathrm{~m}$ placebo $(P<0.05)$ and was $420 \mathrm{~ms}(405 ; 440)$ at $2,048 \mathrm{~m}$ NOT ( $P=\mathrm{NS}$ vs. $2,048 \mathrm{~m}$ placebo). The number of extrabeats and complex arrhythmias was similar over all conditions.

Conclusions: While staying at 2,048 m, lowlanders with COPD experienced nocturnal hypoxemia in association with an increased $\mathrm{HR}$ and prolongation of the QTC interval. NOT significantly improved $\mathrm{SpO}_{2}$ and lowered $\mathrm{HR}$, without changing QTc. Whether oxygen therapy would reduce HR and arrhythmia during longer altitude sojourns remains to be elucidated.

Keywords: cardiac repolarisation, QTC prolongation, heart rate, hypoxia, chronic obstructive pulmonary disease 


\section{INTRODUCTION}

Altitude and air travel for professional and leisure activities is common, exposing millions of people to hypoxemia with potential adverse consequences on their health. Patients with pre-existing chronic obstructive pulmonary disease (COPD) may be at particular risk because of their impaired pulmonary gas exchange even at sea level. In patients with COPD, mortality increases almost linearly with an increasing resting heart rate (HR), with a hazard ratio of 1.39 (95\% CI 1.21-1.60) above $80 \mathrm{bpm}(1,2)$. COPD patients also frequently reveal cardiac repolarization disturbances, including a prolonged QTc interval already near sea level, and this correlated inversely with arterial oxygen saturation $\left(\mathrm{SpO}_{2}\right)$ (3). When exposed to a reduced barometric pressure at altitude or during air travel, patients with COPD are even more hypoxemic than healthy individuals due to impaired pulmonary gas exchange, in particular during sleep (4). Therefore, excessive hypobaric hypoxemia may put COPD patients at risk of an exaggerated HR increase (5) and possibly prolonged QTc and arrhythmias as seen in patients with obstructive sleep apnea (6), although this has not been specifically studied. In the presence of nocturnal hypoxemia and cardiac repolarization disturbances, nocturnal oxygen therapy (NOT) reversed nocturnal hypoxemia and reduced QTc prolongation in patients with pulmonary hypertension (7); however, whether NOT prevents hypoxemia-related cardiac repolarization disturbances in patients with COPD staying overnight at moderate altitude has never been studied.

Therefore, the current study evaluated the hypothesis that, compared with low altitude, sleeping at high altitude increases nocturnal HR and other markers of cardiovascular risks derived from the ECG including the QTc and arrhythmias in lowlanders with COPD and that these effects can be prevented by NOT during the stay at altitude.

\section{METHODS}

\section{Study Design}

This study was part of a randomized, placebo-controlled, crossover trial evaluating the effect of altitude (vs. lowland) and NOT on nocturnal oxygenation and sleep-related breathing disorders in patients with COPD staying at $2,048 \mathrm{~m}$ for 2 consecutive days and nights (ClinicalTrials.gov NCT02150590) (8). The present study focuses on nocturnal HR, cardiac repolarization, and arrhythmia, which have not been addressed previously. The study was conducted from January 1 to October 31,2014 . Patients were studied in Zurich ( $490 \mathrm{~m}$, baseline) and in St. Moritz (2,048 m) during 2 sojourns of 2 days/nights each while receiving either NOT or placebo (sham oxygen and ambient air) according to a randomized, crossover design with a 2-week washout period spent at $<800 \mathrm{~m}$ between stays at $2,048 \mathrm{~m}$. The sleep studies and daytime examinations took place in quiet and comfortable rooms at the University Hospital Zurich and in a mountain hostel in St. Moritz under convenient conditions $(490 \mathrm{~m}$, mean $\pm \mathrm{SD}$ barometric pressure of $722 \pm 2 \mathrm{mmHg}$, temperature of $24.9 \pm 1.0^{\circ} \mathrm{C}$, humidity $42 \pm 5 \%$; and $2,048 \mathrm{~m}$, mean barometric pressure of $595 \pm 3 \mathrm{mmHg}$, temperature of $25.5 \pm 1.6^{\circ} \mathrm{C}$, humidity of $35 \pm 6 \%$ ). Participants gave written informed consent, and the study was approved by the Cantonal Ethics Committee Zurich (EK 2013-0088).

\section{Interventions}

During nights at 2,048 $\mathrm{m}$, either NOT or ambient air (placebo) was administered at a flow rate of $3 \mathrm{~L} / \mathrm{min}$ via nasal cannula. NOT or placebo was delivered via tubing by a concentrator located in a separate room out of the patient's sight (EverFlo, Philips Respironics, Zofingen, Switzerland). During the study, patients continued their usual medication.

For safety reasons, patients experiencing adverse effects at $2,048 \mathrm{~m}$ such as severe hypoxemia $\left(\mathrm{SpO}_{2}<75 \%\right.$ for $\left.>30 \mathrm{~min}\right)$, acute mountain sickness (AMS) diagnosed by an AMSc score $\geq 0.7$ (see below), high blood pressure (systolic $>200 \mathrm{mmHg}$, diastolic $>110 \mathrm{mmHg}$ ), or any other discomfort or intercurrent illness received oxygen and other therapy as appropriate and were evacuated to low altitude at the earliest convenience and withdrawn from the study.

\section{Participants}

COPD patients, living at low altitude $(<800 \mathrm{~m})$, diagnosed according to the Global Initiative for Chronic Obstructive Pulmonary Disease (GOLD) criteria, grades 2-3, 18-75 years of age, both sexes, were recruited among outpatients of the Pulmonary Division, University Hospital Zurich, and surrounding hospitals.

Patients with COPD GOLD grade 4 or 1 , severe gas exchange $\left(\mathrm{SpO}_{2}\right.$ at $490 \mathrm{~m}<92 \%$ and/or $\mathrm{PaCO}_{2}>6 \mathrm{kPa}$ at $\left.490 \mathrm{~m}\right)$, more than mild or inadequately controlled cardiovascular disease, obstructive sleep apnea syndrome, current heavy smoking ( $>20$ cigarettes per day), and previous intolerance to high altitude and stayed at altitude $>1,500 \mathrm{~m}$ for $>2$ days within 4 weeks before the study or pregnancy were excluded from the study.

\section{Measurements and Outcomes}

Clinical examinations and spirometry (9) (Blue Cherry, Geratherm Medical AG, Gschwenda, Germany) were performed. AMS was evaluated by the environmental symptoms cerebral score, which rates 11 questions on symptoms; a value of $\geq 0.7$ in the weighted sum of responses reflects clinically relevant AMS (10). An arterial blood gas analysis was obtained (RapidPoint500, Siemens HealthCare, Zurich, Switzerland) in the morning after sleep studies at $490 \mathrm{~m}$ and at $2,048 \mathrm{~m}$ after NOT and placebo treatment.

During the first night at each location, polysomnography was performed including neurophysiologic standard derivations [2 central electroencephalogram (EEG) leads, electrooculogram (EOG), and electromyogram (EMG)], chest wall excursions by inductance plethysmography, and transcutaneous capnography $\left(\mathrm{PtcCO}_{2}\right)$, pulse oximetry to measure $\mathrm{SpO}_{2}$ and record finger plethysmographic pulse waveforms (PWs) together with a 4channel ECG (leads I-III, aVL, aVR, and aVF) recorded at $200-\mathrm{Hz}$ sampling rate (Alice 5, Philips Respironics, Zofingen, Switzerland) (11). Mean nocturnal $\mathrm{SpO}_{2}$, the number of oxygen desaturation events per hour [the oxygen desaturation index (ODI), >3\% dips], apnea/hypopnea, and sleep stages were scored according to international standards. 
Time series of overnight recordings of ECG and PW were processed by custom-built MATLAB program during the first night at 490 and 2,048 $\mathrm{m}$ under NOT or placebo. The software automatically detected the $\mathrm{R}$ wave of each QRS complex and ensemble averaged the ECG and corresponding PW cycles over successive 1-min intervals throughout the night. From ECG lead II (or lead I in case of artifacts), the QT interval was measured from the earliest onset of the $\mathrm{Q}$ wave until the intersection point between the isoelectric line and the tangent of the downslope of the $\mathrm{T}$ wave (Figure 1). HRadjusted QT intervals (QTc), computed by Bazett's formula [QTc (ms) = QT (ms)/ $\sqrt{ }$ RR interval (s)] (12), were determined minute by minute and overnight means, and 90th and 95th percentiles of 1-min means of QTc computed. In PW curves, the peak-to-peak time [PPT (ms), from systolic maximum to diastolic maximum or inflection point], the stiffness index

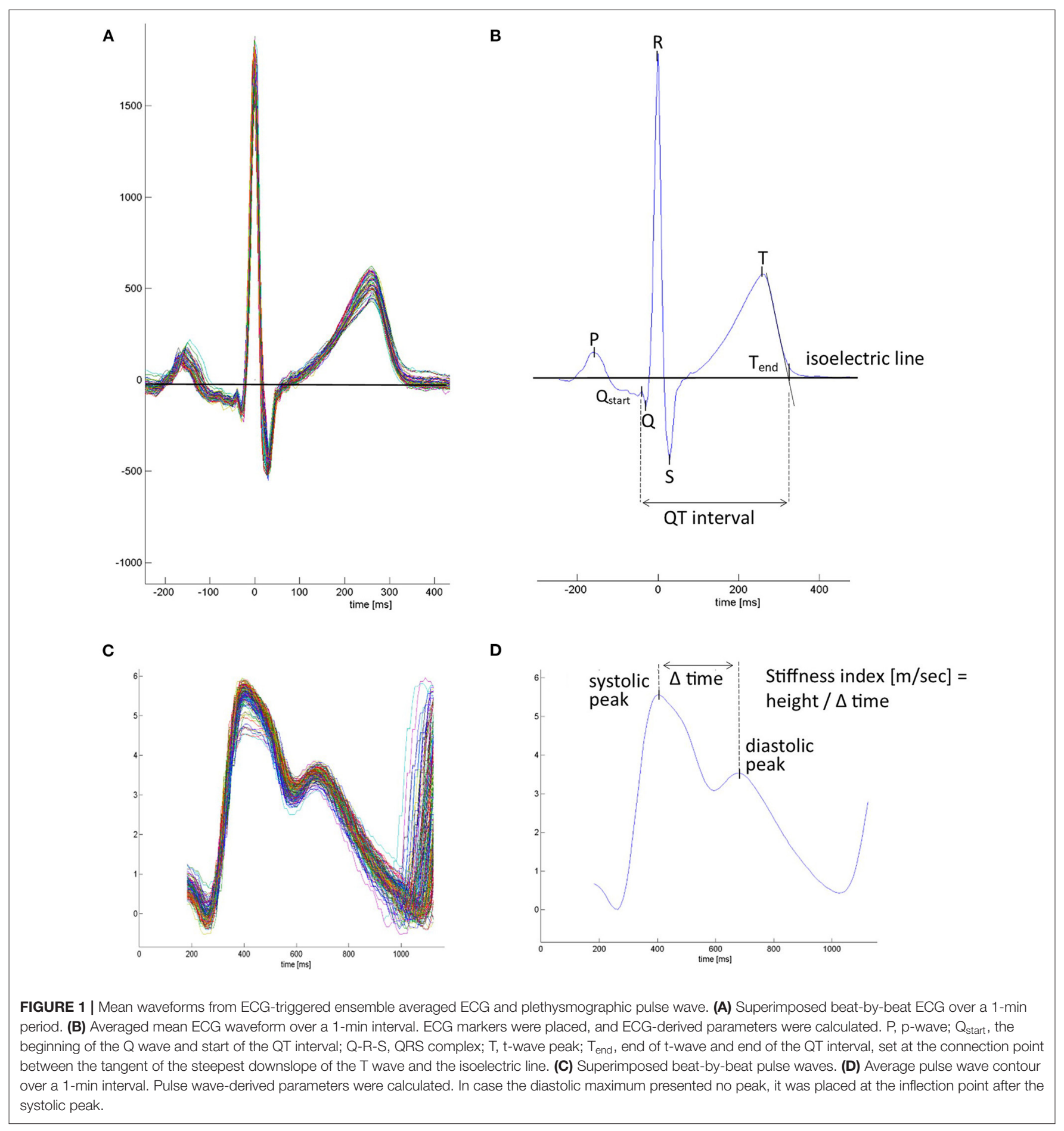


[SI $(\mathrm{m} / \mathrm{s})$, body height divided by PPT] $(13,14)$, and the pulse transit time (PTT) measured from the ECG R-peak to the time at 50\% PW amplitude were determined (Figure 1) and calculated. Arrhythmic events defined as supraventricular and ventricular extrasystoles (SVES and VES), and complex arrhythmias [CAs; bigeminy, trigeminy, couplet, and triplets and ventricular tachycardia (if any were recorded)] were manually scored. Blood pressure was measured in the evening and morning after $15 \mathrm{~min}$, with the patient in an awake, relaxed, and supine position with a sphygmomanometer device.

The main outcomes of the current study were the QTc and other variables derived from the ECG and PW analyses, clinical evaluations, questionnaires, and results from sleep studies during the first night at each location. A separate sample size estimation was not performed for this study, as the number of required participants was determined for the main trial.

\section{Randomization and Blinding}

Participants were randomized in balanced blocks of 4 by drawing an envelope containing 1 of 4 sequences of altitude exposure and treatment: (A) $490 \mathrm{~m}-2,048 \mathrm{~m}$ placebo-2,048 m
NOT; (B) $490 \mathrm{~m}-2,048 \mathrm{~m}$ NOT-2,048 m placebo; (C) 2,048 m placebo-2,048 $\mathrm{m}$ NOT-490 m; and (D) 2,048 $\mathrm{m}$ NOT-2,048 m placebo- $490 \mathrm{~m}$. Patients and assessors were blinded to the treatment until conclusion of data analysis.

\section{Data Analysis and Statistics}

Data were analyzed by the per-protocol approach including data from all participants completing the first night at $490 \mathrm{~m}$ and 2,048 $\mathrm{m}$ under NOT and placebo. Results are presented as median (quartiles). To evaluate overall effects between $490 \mathrm{~m}$, 2,048 m placebo, and 2,048 $\mathrm{m}$ NOT, repeated-measures ANOVA was performed followed by paired $t$-test or Wilcoxon signed rank test. Multivariable regression analysis was used to evaluate the effects of altitude and NOT on outcomes while controlling for elapsed time and baseline variables. A probability of $P<0.05$ was considered statistically significant.

\section{RESULTS}

Forty-two patients were assessed for eligibility, and 10 were excluded for various reasons (Figure 2). At 2,048 $\mathrm{m}$ under

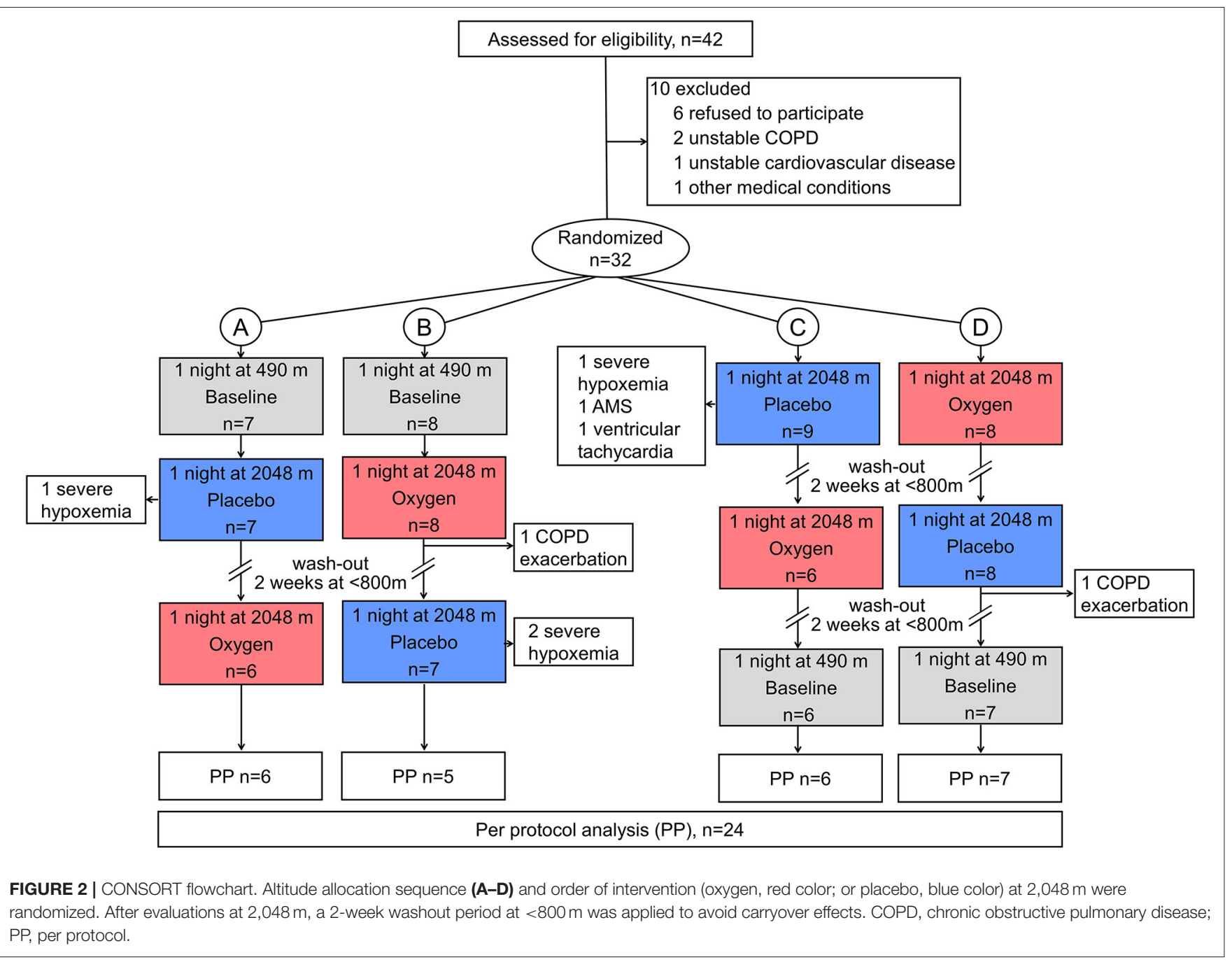


placebo therapy, 8 patients had to be withdrawn from the study because of adverse events, including severe hypoxemia $(n=4$, $\mathrm{SpO}_{2}<75 \%$ for $\left.>30 \mathrm{~min}\right)$, COPD exacerbation $(n=2)$, AMS $(n=1)$, and nocturnal non-sustained ventricular tachycardia $(n=1)$. The patient experiencing nocturnal ventricular tachycardia (mean nocturnal QTc prolongation of $448 \mathrm{~ms}$ ) was referred to a cardiologist and started beta-blocker therapy. One additional patient completed all examinations and was included in the final analysis; however, he experienced a panic attack at 2,048 $\mathrm{m}$ after placebo. At 2,048 $\mathrm{m}$ under NOT, one patient experienced a COPD exacerbation. As previously published, NOT significantly reduced the proportion of altitude-related illnesses compared with placebo intervention ( 8 vs. $1, P<0.001$ ) (15). At 2,048 m, patients experiencing an altitude-related illness were treated with oxygen and medication as appropriate and were relocated to low altitude, which led to recovery without sequelae. Data of 24 patients, 11 men and 13 women, were analyzed (Figure 2). Participant characteristics are presented in Table $\mathbf{1 .}$

The main results are summarized in Table 2 and illustrated in Figures 3, 4. With altitude exposure using placebo, there was a significant increase in nocturnal $\mathrm{HR}$ and prolongation of the mean, and the 90th and 95th percentiles of QTc. At $490 \mathrm{~m}$ and $2,048 \mathrm{~m}$ under placebo therapy, 1 of 24 (4\%) patients had QTc prolongations exceeding the clinically relevant threshold of $>450 \mathrm{~ms}$ in men or $>460 \mathrm{~ms}$ in women compared with 0 of 24 patients while staying at 2,048 $\mathrm{m}$ under NOT $(P=$ NS).

TABLE 1 | Patient characteristics.

\begin{tabular}{lc}
\hline Variables & \\
\hline$n$ & $24(11$ men, 13 women) \\
Age, years & $66(63 ; 70)$ \\
Body mass index, $\mathrm{kg} / \mathrm{m}^{2}$ & $25.1(21.4 ; 28.6)$ \\
FEV,$\%$ predicted & $55(40 ; 62)$ \\
Smoking, pack-years & $40(26 ; 58)$ \\
Current smoker, $n$ (\%) & $10(42)$ \\
COPD GOLD, grade 2/grade 3, $n$ & $17 / 7$ \\
Comorbidities, $\boldsymbol{n}$ (\%) & \\
Cardiovascular disease including hypertension & $13(54)$ \\
Diabetes & $3(13)$ \\
Depression & $3(13)$ \\
Medication, $\boldsymbol{n}$ (\%) & \\
Inhaled glucocorticosteroids & $2(8)$ \\
Inhaled $\beta$-adrenergics & $18(75)$ \\
Inhaled anticholinergics & $19(79)$ \\
Diuretics & $2(8)$ \\
Antihypertensive medication & $13(54)$ \\
$\beta$-Blockers & $1(4)$ \\
Antidiabetics & $3(13)$ \\
Antidepressants & $3(13)$ \\
\hline
\end{tabular}

Data presented as median (quartiles) and numbers (n).

FEV 1 , forced expiratory volume in 1s; COPD GOLD, Global Initiative for Chronic Obstructive Pulmonary Disease.
The mean nocturnal $\mathrm{SpO}_{2}$ was significantly lower at 2,048 m placebo compared with $490 \mathrm{~m}$ and $2,048 \mathrm{~m}$ NOT $(P<0.05$ both comparisons), and $\mathrm{SpO}_{2}$ at 2,048 $\mathrm{m}$ NOT was higher compared with $490 \mathrm{~m}(P<0.05$, Figure $3 \mathrm{C})$. The ODI was $3.6 / \mathrm{h}(1.3 ; 13.7)$ at $490 \mathrm{~m}, 37.8 / \mathrm{h}(16.7 ; 59.5)$ at $2,048 \mathrm{~m}$ placebo, and $0.6 / \mathrm{h}(0.1$; $2.6)$ at $2,048 \mathrm{~m}$ NOT $(P<0.05$ all comparisons, Figure 3D). The mean nocturnal $\mathrm{HR}$ was higher at $2,048 \mathrm{~m}$ placebo compared with $490 \mathrm{~m}$ and $2,048 \mathrm{~m}$ NOT $(P<0.05$ both comparisons, Figure 3A). The PTT was reduced at $2,048 \mathrm{~m}$ placebo compared with $490 \mathrm{~m}$ and $2,048 \mathrm{~m}$ NOT $(P<0.05$ both comparisons), and SI remained unchanged at $490 \mathrm{~m}, 2,048 \mathrm{~m}$ placebo, and 2,048 $\mathrm{m}$ NOT. QTc was prolonged at 2,048 $\mathrm{m}$ placebo compared with $490 \mathrm{~m}$ (Table 2, Figure 3B). NOT significantly reduced nocturnal $\mathrm{HR}$, lowered the QT but did not modify the QTc interval, lowered $\mathrm{ODI}$, and increased $\mathrm{SpO}_{2}$ at 2,048 $\mathrm{m}$ compared with placebo (Figure 4). No change in the number of arrhythmic events (VES, SVES, and CA) was observed at altitude with placebo or NOT compared with $490 \mathrm{~m}$ (Table 2).

Multivariable regression analysis of consecutive 1-min means of QTc revealed that altitude was an independent predictor of an increased QTc; furthermore, it revealed that QTc increased in the early night and reversed to awake QTc values in the early morning hours of the night, independent of altitude or treatment and when controlled for age, sex, and forced expiratory volume in $1 \mathrm{~s}\left(\mathrm{FEV}_{1}\right) \%$ predicted (Table 3, Figure 5).

\section{DISCUSSION}

This randomized, placebo-controlled crossover trial in patients with moderate to severe COPD demonstrated that sleeping at 2,048 $\mathrm{m}$, an altitude of many tourist destinations and permanent settlements, is associated with hypoxemia, an increased nocturnal $\mathrm{HR}$, a reduced PTT, and a moderate prolongation of the QTc interval. Reassuringly, a QTc prolongation above the limit considered clinically relevant was observed in only one patient (already at low altitude), and symptomatic arrhythmias were recorded in only 1 other patient. However, 8 (26\%) of the COPD patients suffered from altitude-related illnesses requiring medical intervention and descent to lower altitude. NOT significantly reduced the incidence of altitude-related illnesses at $2,048 \mathrm{~m}$ compared with placebo, improved the $\mathrm{SpO}_{2}$, mitigated the altitude-induced increase in $\mathrm{HR}$, and prevented the reduction in PTT but did not change the QTc.

A higher resting HR is a strong and independent predictor of mortality in patients with COPD with the relative risk increasing by $21 \%$ by every $10 \mathrm{bpm}$ (1). A HR $>80 \mathrm{bpm}$ was associated with a significantly increased all-cause mortality compared with $<80 \mathrm{bpm}$ (hazard ratio 1.6, 95\% CI 1.1-2.3). Comparably, in a large cohort of COPD patients at cardiac risk, resting HR above $80 \mathrm{bpm}$ revealed a hazard ratio of 1.39 (1.21-1.60) (2). In the present study, we found a significant increase in mean nocturnal $\mathrm{HR}$ of, on average, $6 \mathrm{bpm}$ when COPD patients traveled from 490 to $2,048 \mathrm{~m}$. The resulting resting HR was 82 bpm, a level that may well be associated with adverse health events in the long run (1). Prospective long-term trials in patients with COPD staying at high altitude are required to elucidate 
TABLE 2 | Main outcomes derived from sleep studies during the first night and the following morning at each location.

\begin{tabular}{|c|c|c|c|c|}
\hline Variables & $490 \mathrm{~m}$ & 2,048 m placebo & $2,048 \mathrm{~m}$ NOT & $P$, ANOVA \\
\hline \multicolumn{5}{|l|}{ ECG analysis } \\
\hline Heart rate, bpm & $73(66 ; 82)$ & $82(71 ; 85)^{\star \pi}$ & $78(67 ; 84)$ & $<0.001$ \\
\hline QT duration, ms & $380(365 ; 410)$ & $375(355 ; 390)^{* \pi}$ & $390(370 ; 400)$ & $<0.001$ \\
\hline QTc duration, ms & $417(404 ; 439)$ & $426(405 ; 440)^{\star}$ & $420(405 ; 440)$ & 0.028 \\
\hline 90th percentile QTc, ms & $422(410 ; 436)$ & $430(406 ; 445)^{\star}$ & $427(405 ; 446)$ & 0.016 \\
\hline 95th percentile QTc, ms & $424(412 ; 438)$ & $432(408 ; 447)^{\star}$ & $430(408 ; 447)$ & 0.024 \\
\hline QTc max, ms & $439(425 ; 455)$ & $444(418 ; 457)$ & 442 (420; 452) & 0.397 \\
\hline TpTe, ms & $60(55 ; 70)$ & $60(55 ; 75)$ & $65(60 ; 70)$ & 0.581 \\
\hline TpTe c, ms & $65(62 ; 73)$ & $68(60 ; 79)$ & $71(61 ; 80)$ & 0.836 \\
\hline VES, 1/h & $0.3(0.0 ; 2.4)$ & $1.6(0.1 ; 14.5)$ & $0.3(0.1 ; 2.9)$ & 0.341 \\
\hline SVES, $1 / \mathrm{h}$ & $1.8(0.4 ; 10.0)$ & $2.4(0.4 ; 7.8)$ & $2.2(0.5 ; 7.5)$ & 0.362 \\
\hline Complex arrhythmia, 1/h & $0(0 ; 0)$ & $0(0 ; 0)$ & $0(0 ; 0)$ & 0.249 \\
\hline \multicolumn{5}{|l|}{ Pulse wave analysis } \\
\hline Peak-to-peak time, ms & $160(150 ; 180)$ & $160(153 ; 178)$ & $170(160 ; 190)$ & 0.559 \\
\hline Stiffness index, m/s & $10.2(9.2 ; 11.1)$ & $10.5(9.2 ; 11.1)$ & $10.1(8.9 ; 10.5)$ & 0.388 \\
\hline Pulse transit time, ms & $345(337 ; 364)$ & $332(325 ; 351)^{* \pi}$ & $352(338 ; 365)$ & $<0.001$ \\
\hline Reflection index & $0.74(0.68 ; 0.79)$ & $0.75(0.62 ; 0.76)^{\star}$ & $0.75(0.65 ; 0.78)$ & 0.034 \\
\hline \multicolumn{5}{|c|}{ Nocturnal oxygenation and capnography } \\
\hline Mean nocturnal $\mathrm{SpO}_{2}, \%$ & $92(90 ; 94)$ & $86(83 ; 89)^{\star \star \pi}$ & $97(95 ; 98)^{\star}$ & $<0.001$ \\
\hline ODI, 1/h & $3.6(1.3 ; 13.7)$ & $37.8(16.7 ; 59.5)^{* \pi}$ & $0.6(0.1 ; 2.6)^{\star}$ & $<0.001$ \\
\hline $\mathrm{PtcCO}_{2}, \mathrm{mmHg}$ & $41(37 ; 46)$ & $44(42 ; 48)^{\star \star \pi}$ & $48(42 ; 52)^{\star}$ & $<0.001$ \\
\hline \multicolumn{5}{|l|}{ Morning evaluation } \\
\hline BP systolic, mmHg & $122(111 ; 135)$ & $125(112 ; 137)$ & $125(116 ; 134)$ & 0.679 \\
\hline BP diastolic, mmHg & $70(65 ; 77)$ & $69(62 ; 82)$ & $69(65 ; 80)$ & 0.762 \\
\hline $\mathrm{BP}$ mean, mmHg & $85(80 ; 94)$ & $86(78 ; 98)$ & $86(82 ; 98)$ & 0.695 \\
\hline \multicolumn{5}{|l|}{ Arterial blood gases } \\
\hline $\mathrm{pH}$ & $7.44(7.43 ; 7.46)$ & $7.47(7.45 ; 7.49)^{\star}$ & $7.47(7.45 ; 7.48)^{*}$ & $<0.001$ \\
\hline $\mathrm{PaCO}_{2}, \mathrm{kPa}$ & $5.1(4.7 ; 5.2)$ & $4.7(4.2 ; 7.9)^{\star}$ & $4.5(4.0 ; 5.0)^{\star}$ & $<0.001$ \\
\hline $\mathrm{PaO}_{2}, \mathrm{kPa}$ & $9.0(8.3 ; 9.8)$ & $8.1(7.3 ; 8.6)^{\star}$ & $8.0(7.2 ; 8.4)^{\star}$ & $<0.001$ \\
\hline $\mathrm{SaO}_{2}, \%$ & $94.4(92.5 ; 95.3)$ & $90.1(87.7 ; 92.1)^{\star}$ & $90.6(87.6 ; 91.8)^{*}$ & $<0.001$ \\
\hline
\end{tabular}

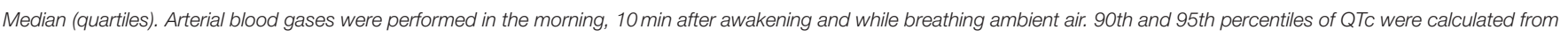
the average 1-min QTC values over the complete night.

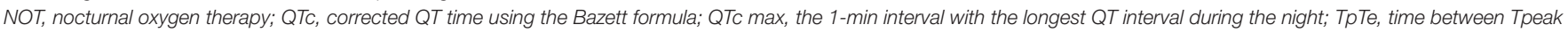

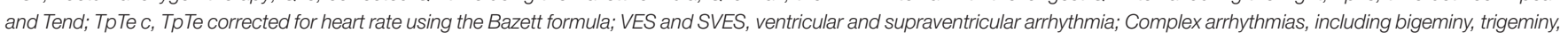

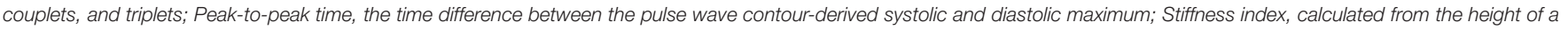

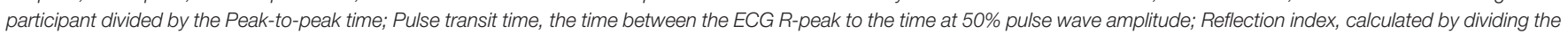

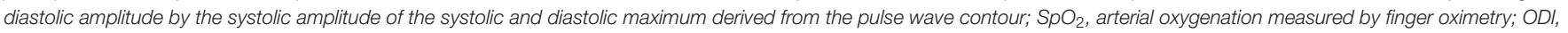
oxygen desaturation index defined as number of $>3 \%$ dips in $\mathrm{SpO}_{2} ; \mathrm{PtcCO}_{2}$, transcutaneous carbon dioxide; $\mathrm{BP}$, blood pressure.

${ }^{\star} P<0.05$ vs. $490 \mathrm{~m} ; \boldsymbol{9} P<0.05$ vs. NOT.

whether the observed altitude-related HR increase represents a similar hazard as if it was developing at low altitude or whether it is a beneficial adaptation to hypoxia. The HR increase with altitude in the present COPD cohort was doubled compared with healthy exposed to even slightly higher altitudes of 2,590 m (16), and the findings confirm the exaggerated HR increase in COPD patients exposed to isocapnic hypoxia (5). When the present COPD patients collective used NOT at altitude, the mean nocturnal HR increase could be completely reversed to lowaltitude levels $<80 \mathrm{bpm}$, a reduction that can be considered relevant to decreased cardiovascular risk in the long run $(1,2)$. Consistent with an increased sympathetic excitation, the PTT was reduced at 2,048 $\mathrm{m}$ under placebo but not under NOT therapy.
In the general population, a prolonged QTc has been found to be a risk factor for ventricular fibrillation and sudden cardiac death $(17,18)$, although arrhythmias are more often associated with an even longer QTc of $>500 \mathrm{~ms}$ (17). A clinically important threshold for QTc prolongation has been suggested to be $>450 \mathrm{~ms}$ in men and $>460 \mathrm{~ms}$ in women $(19,20)$. The values observed in the current COPD patients were well below this threshold at low and high altitudes; however, at moderate altitude, QTc might have been prolonged due to altitude-induced hypoxemia and its consequences on the cardiovascular and ventilatory system and possibly other, unknown factors.

It has been shown that COPD is associated with cardiac arrhythmias and specific ECG alterations that have prognostic 


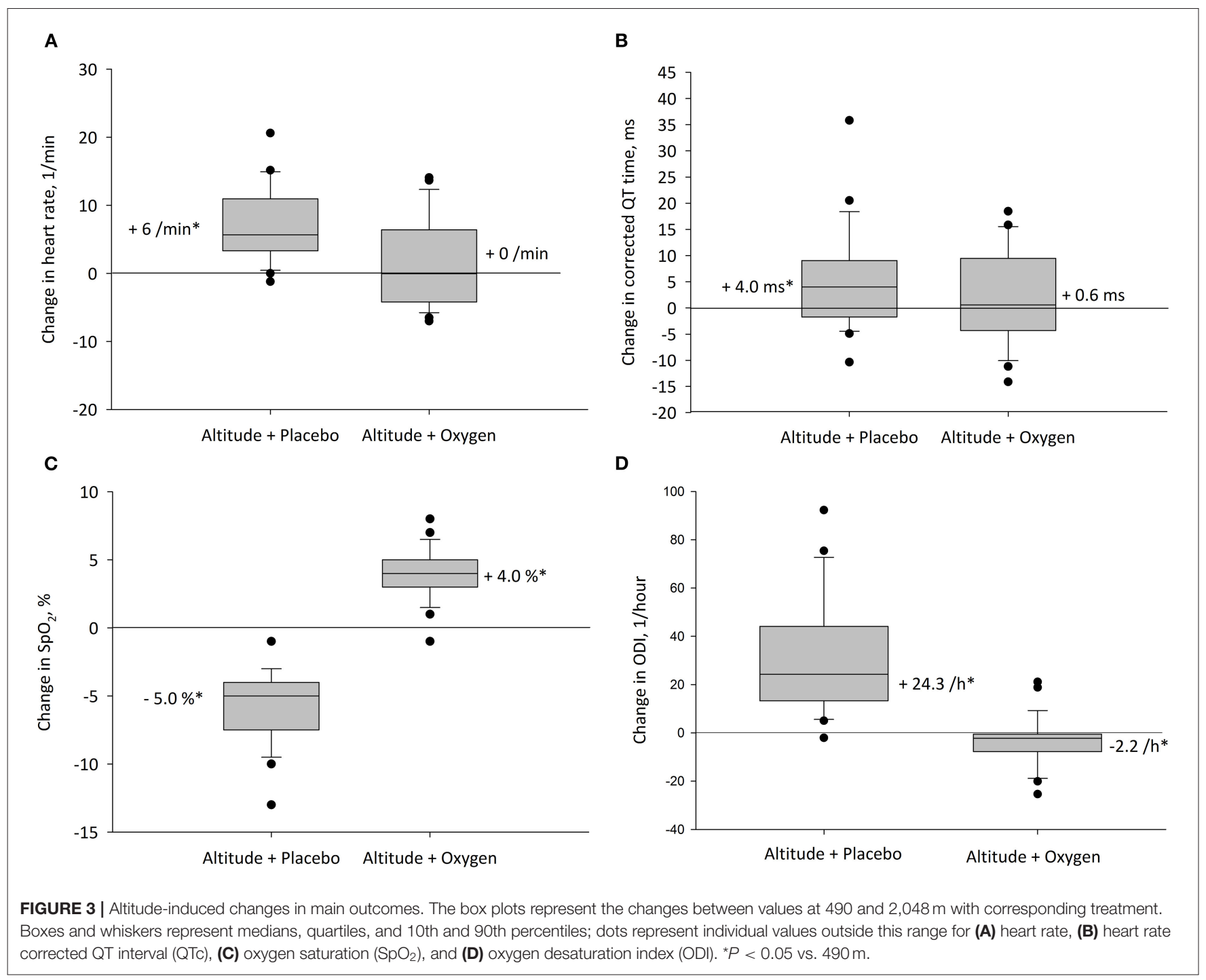

significance (21). The prevalence of QTc prolongation in COPD patients in comparison with the general population (22) remains controversial since it has been suggested to be increased (3), reduced (23), or similar (24). In the currently studied cohort of mildly hypoxemic and non-hypercapnic patients with COPD, a low prevalence of clinically important prolongations in QTc was observed at low altitude. The mean QTc of the night exceeded the upper limit of normal in no patient, and the 95th percentile of nocturnal QTc exceeded the upper limit of normal in 2 of 24 patients. In a prospective 5-year cohort study including 246 patients with COPD, prolonged QTc and other QT-derived parameters were found to be predictors of poor survival (25). The association with prolonged cardiac repolarization and sudden cardiac death in COPD patients is however not yet confirmed and needs further investigation (3). Tirlapur and Mir (26) recorded nocturnal electrocardiographic changes in 12 severely hypoxemic and hypercapnic COPD patients at low altitude. In several of them, major QTc prolongations were noted, and these were improved by supplemental oxygen administration.
Consistent with these observations, we found that aggravation of hypoxemia during exposure to hypobaric hypoxia at high altitude was associated with a QTc prolongation in the studied patients with COPD, which were mildly hypoxemic and nonhypercapnic. We can therefore confirm that altitude induced nocturnal hypoxemia and prolonged QTc; however, along with a significant $\mathrm{HR}$ reduction, we found no modification in the already relatively low QTc under NOT (27).

A previous study in COPD patients (28) and a recent metaanalysis (29) suggest that prolongation in the Tpeak to Tend intervals may predict sudden cardiac death. In the current study, Tpeak to Tend did not change with altitude exposure possibly because of a reduced sensitivity of this variable to hypoxemia.

COPD has been associated with a higher prevalence of cardiac arrhythmias, angina, acute myocardial infarction, congestive heart failure, stroke, pulmonary embolism, and an increased risk of death due to cardiovascular disease (30). In the current study, we found numerous short, self-limited arrhythmic events, mainly pre-mature beats, which were not altered by sleeping at high 


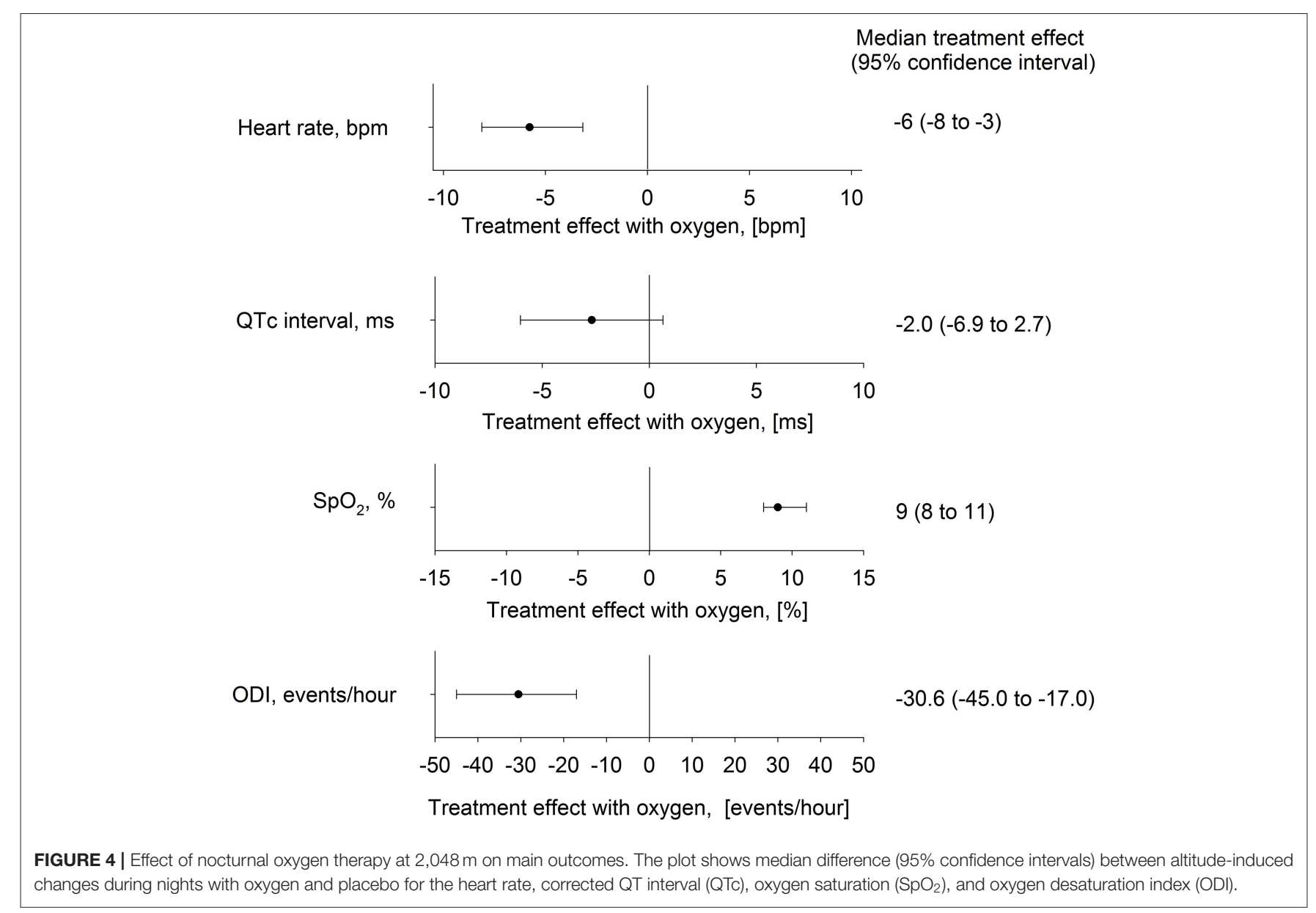

altitude, and thus our findings did not confirm some previous studies $(26,31,32)$. We found no relevant sustained arrhythmias at both altitude with and without NOT; however, one patient had three runs of ventricular tachycardia during the night at 2,048 m with placebo therapy. This patient was referred to a cardiologist and commenced beta-blocker therapy.

The literature on ECG recordings during real or simulated altitude is scarce. In 8 COPD patients investigated at $1,920 \mathrm{~m}$, no cardiac irregularity during daytime ECG recordings was observed (33). In studies evaluating the exposure of COPD patients to aircraft cabin conditions at $2,438 \mathrm{~m}$ altitude equivalent, no change in arrhythmias or ischemia signs (34) except for isolated SVES and VES (35) was found. However, no further information about the ECG characteristics at baseline was described. Gong et al. (36) studied the respiratory and cardiac response to altitude in 22 COPD patients. By giving the participants hypoxic gas mixtures corresponding to $1,524,2,438$, and $3,048 \mathrm{~m}, 10$ out of $22(45.5 \%)$ COPD patients developed asymptomatic cardiac arrhythmias, consisting of SVES and VES. Oxygen administration improved almost all physiologic indexes. In the present study, we found no relevant sustained arrhythmias at both altitudes with and without NOT. As QTc prolongation persisted despite the correction of nocturnal hypoxemia by NOT, other factors than hypoxemia associated
TABLE 3 | Night-time progression of QTC: mixed, linear regression analysis.

\begin{tabular}{|c|c|c|c|c|}
\hline Dependent variable: QTc, ms & Coefficient & SE & $95 \% \mathrm{Cl}$ & $P$ \\
\hline \multicolumn{5}{|l|}{490 m (ref) } \\
\hline $2,048 \mathrm{~m}$ placebo vs. $490 \mathrm{~m}$ & 4.7 & 0.1 & 4.5 to 4.9 & $<0.001$ \\
\hline 2,048 m NOT vs. $490 \mathrm{~m}$ & 1.8 & 0.1 & 1.6 to 2.0 & $<0.001$ \\
\hline \multicolumn{5}{|l|}{ Night-time } \\
\hline \multicolumn{5}{|l|}{ First 10 min (ref) } \\
\hline $11-70 \mathrm{~min}$ & 3.6 & 0.3 & 3.0 to 4.2 & $<0.001$ \\
\hline $71-130 \mathrm{~min}$ & 4.0 & 0.3 & 3.4 to 4.6 & $<0.001$ \\
\hline $131-190 \mathrm{~min}$ & 3.3 & 0.3 & 2.6 to 3.9 & $<0.001$ \\
\hline $191-250 \mathrm{~min}$ & 3.2 & 0.3 & 2.6 to 3.8 & $<0.001$ \\
\hline 251-310 min & 2.8 & 0.3 & 2.2 to 3.4 & $<0.001$ \\
\hline $311-370 \mathrm{~min}$ & 0.9 & 0.3 & 0.3 to 1.5 & 0.003 \\
\hline $371-430 \mathrm{~min}$ & 0.1 & 0.3 & -0.5 to 0.7 & 0.739 \\
\hline $431-480 \mathrm{~min}$ & -1.0 & 0.3 & -1.6 to -0.4 & 0.002 \\
\hline Age, years & 0.9 & 1.4 & -1.8 to 3.6 & 0.504 \\
\hline Sex, male vs. female & -17.2 & 15.5 & -47.6 to 13.2 & 0.267 \\
\hline $\mathrm{FEV}_{1} \%$ pred. at $490 \mathrm{~m}$ & -0.3 & 0.6 & -1.5 to 0.9 & 0.664 \\
\hline Intercept & 374.4 & 110.3 & 158.2 to 590.5 & 0.001 \\
\hline
\end{tabular}

The dependent variable QTC was averaged over consecutive 1-min intervals throughout the nights, and a total of 31,589 1-min intervals were included in this mixed, linear regression analysis.

FEV 1 , forced expiratory volume in the first second of expiration. 


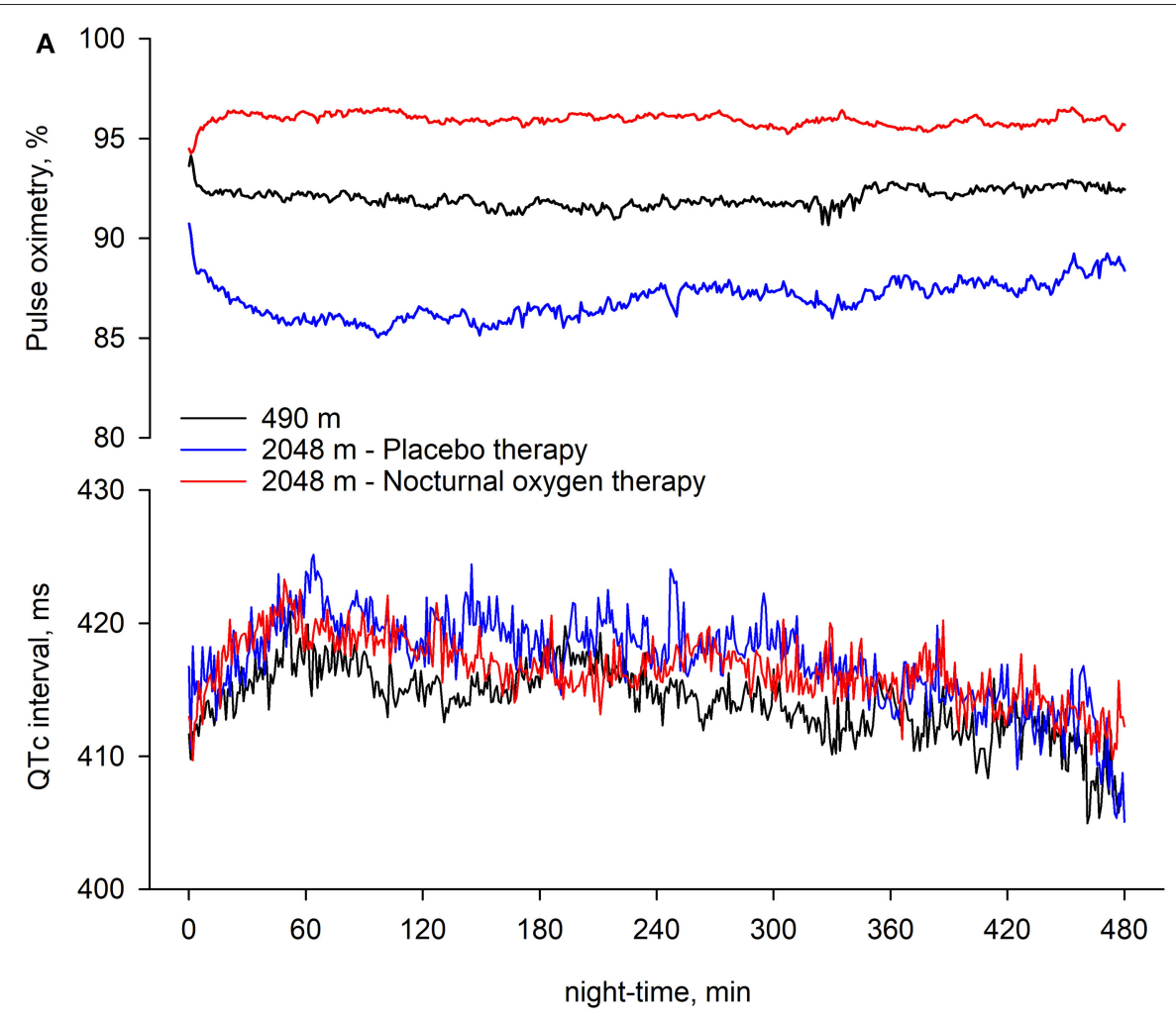

B

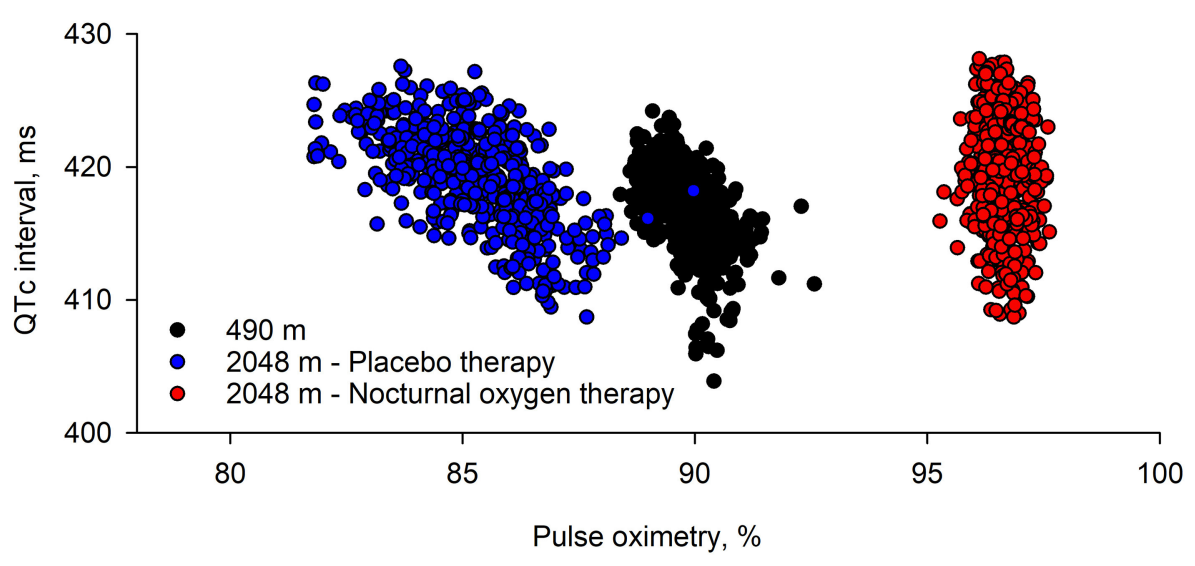

FIGURE 5 | Nocturnal pulse oximetry and corrected QT interval in relation to the underlying intervention. Time series of group means of pulse oximetry and corrected QT interval (QTC) over consecutive 1-min periods during study nights are displayed. At $490 \mathrm{~m}$, arterial oxygen saturation shortly decreases after lights off and remained lower until the end of the night. At 2,048 m under placebo therapy, arterial oxygen saturation shortly decreases after lights off and steadily improves over the night. At 2,048 m under nocturnal oxygen therapy, arterial oxygen saturation increases and remains higher until the end of the night. Compared with $490 \mathrm{~m}$, at 2,048 $\mathrm{m}$ under placebo therapy, QTc remains elevated throughout the night; however, independent of the intervention, QTc increases in the $1 \mathrm{~h}$ of the night, while it progressively returns to lower values thereafter.

with the stay at high altitude including alterations in intravascular volume, $\mathrm{pH}$, and serum electrolyte concentrations (calcium and potassium) and further, unknown causes might have affected cardiac repolarization $(37,38)$.

Furthermore, we performed pulse wave analysis and assessed the arterial stiffness index SI, a marker previously shown to be associated with cardiovascular risk (14). Thus, the study by
Clarenbach et al. showed a SI of $9.4 \mathrm{~m} / \mathrm{s}$ in patients with 2 or more cardiovascular risk factors compared with $6.4 \mathrm{~m} / \mathrm{s}$ in a control group without any cardiovascular risk factors. Our results confirm that COPD patients have an elevated SI of 10.2 $\mathrm{m} / \mathrm{s}$ already at $490 \mathrm{~m}$; however, moderate altitude and NOT intervention did not further change the SI, suggesting a low sensitivity to acute hypoxemia. 
The limitations of our study are the relatively small sample size and the short exposure time to altitude. However, the randomized, crossover design allowed to reduce sample size, as each patient served as his/her own control. This study applied 3 $\mathrm{L} / \mathrm{min}$ of NOT through a nasal cannula to improve hypoxemia and sleep-disturbed breathing despite possible mouth breathing. This intervention improved $\mathrm{SpO}_{2}$ and ODI (Table 2) beyond the values obtained at $490 \mathrm{~m}$, suggesting that an even lower dose of NOT might be sufficient during altitude travel in certain patients. Investigating cardiac rhythms and repolarization during longer altitude stays would be interesting but was logistically not feasible. Patients with more severe COPD, exacerbation, or severe daytime hypoxia were not included in this trial, and thus further studies at higher altitudes and in patients with more severe COPD are necessary to widen the knowledge of effects of sleeping at altitude in more severe COPD on HR and indices of repolarization.

\section{CONCLUSIONS}

This randomized, placebo-controlled crossover trial on the effect of a stay at altitude $(2,048 \mathrm{~m})$ in COPD patients on cardiac rhythm and repolarization revealed a significant increase in mean nocturnal HR and a prolongation of QTc within the normal range. Cardiac arrhythmias were generally uncommon and clinically relevant, requiring descent to lower altitude in only 1 of the 24 patients. Nevertheless, several patients experienced other altitude-related adverse effects on their health that were effectively prevented by NOT. Therefore, patients with moderate to severe COPD might consider using NOT during altitude sojourns.

\section{REFERENCES}

1. Warnier MJ, Rutten FH, de Boer A, Hoes AW, De Bruin ML. Resting heart rate is a risk factor for mortality in chronic obstructive pulmonary disease, but not for exacerbations or pneumonia. PLoS ONE. (2014) 9:e105152. doi: 10.1371/journal.pone.0105152

2. Byrd JB, Newby DE, Anderson JA, Calverley PMA, Celli BR, Cowans NJ, et al. Blood pressure, heart rate, and mortality in chronic obstructive pulmonary disease: the SUMMIT trial. Eur Heart J. (2018) 39:3128-34. doi: 10.1093/eurheartj/ehy451

3. Sievi NA, Clarenbach CF, Camen G, Rossi VA, van Gestel AJ, Kohler M. High prevalence of altered cardiac repolarization in patients with COPD. BMC Pulm Med. (2014) 14:55. doi: 10.1186/1471-2466-14-55

4. Luks AM, Swenson ER. Travel to high altitude with pre-existing lung disease. Eur Respir J. (2007) 29:770-92. doi: 10.1183/09031936.00052606

5. Miyamoto K, Nishimura M, Akiyama Y, Yamamoto H, Kishi F, Kawakami Y. Augmented heart rate response to hypoxia in patients with chronic obstructive pulmonary disease. Am Rev Respir Dis. (1992) 145:1384-8. doi: 10.1164/ajrccm/145.6.1384

6. Latshang TD, Kaufmann B, Nussbaumer-Ochsner Y, Ulrich S, Furian M, Kohler M, et al. Patients with obstructive sleep apnea have cardiac repolarization disturbances when travelling to altitude: randomized, placebo-controlled trial of acetazolamide. Sleep. (2016) 39:1631-7. doi: $10.5665 /$ sleep. 6080

7. Schumacher DS, Müller-Mottet S, Hasler ED, Hildenbrand FF, Keusch S, Speich R, et al. Effect of oxygen and acetazolamide on nocturnal cardiac conduction, repolarization, and arrhythmias in precapillary pulmonary

\section{DATA AVAILABILITY STATEMENT}

The datasets presented in this article are not readily available because the raw data supporting the conclusions of this article include sensitive patient data. Requests to access the datasets should be directed to michael.furian@usz.ch.

\section{ETHICS STATEMENT}

This study involved human participants and was reviewed and approved by Cantonal Ethics Committee Zurich (EK-20130088). The patients/participants provided their written informed consent to participate in this study.

\section{AUTHOR CONTRIBUTIONS}

MB and MF had full access to all the data in the study and take responsibility for the integrity of the data and the accuracy of the data analysis. KB, TL, SU, and MF: concept and design. All authors: acquisition, analysis, or interpretation of data, and critical revision of the manuscript for important intellectual content. $\mathrm{MB}, \mathrm{KB}$, and MF: drafting of the manuscript and statistical analysis. KB: obtained funding. KB, TL, SA, FH, DF, SU, and MF: administrative, technical, or material support and supervision.

\section{FUNDING}

This study was supported by the Swiss National Science Foundation and Lunge Zurich. Siemens Health Engineers provided some equipment for the study.

hypertension and sleep-disturbed breathing. Chest. (2014) 146:1226-36. doi: $10.1378 /$ chest.14-0495

8. Tan L, Furian M, Latshang TD, Aeschbacher SS, Huber F, Flueck $\mathrm{D}$, et al. Late breaking abstract - effect of oxygen supplementation on sleep and nocturnal breathing in patients with chronic obstructive pulmonary disease travelling to high altitude: randomized trial. Eur Respir J. (2018) 52(Suppl 62):PA3339. doi: 10.1183/13993003.congress-2018. PA3339

9. Levy ML, Quanjer PH, Booker R, Cooper BG, Holmes S, Small I, et al. Diagnostic spirometry in primary care: proposed standards for general practice compliant with American thoracic society and European respiratory society recommendations: a general practice airways group (GPIAG)1 document, in association with the association for respiratory technology \& physiology (ARTP)2 and education for Health3 1 www.gpiag.org 2 www.artp.org 3 www.educationforhealth.org.uk. Prim Care Respir J. (2009) 18:130-47. doi: 10.4104/pcrj.2009. 00054

10. Sampson JB, Cymerman A, Burse RL, Maher JT, Rock PB. Procedures for the measurement of acute mountain sickness. Aviat Space Environ Med. (1983) 54:1063-73.

11. Latshang TD, Nussbaumer-Ochsner Y, Henn RM, Ulrich S, Lo Cascio CM, Ledergerber B, et al. Effect of acetazolamide and autoCPAP therapy on breathing disturbances among patients with obstructive sleep apnea syndrome who travel to altitude: a randomized controlled trial. JAMA. (2012) 308:23908. doi: 10.1001/jama.2012.94847

12. Bazett H. An analysis of the time-relations of electrocardiograms. Heart. (1920) 7:353-70. 
13. Millasseau SC, Kelly RP, Ritter JM, Chowienczyk PJ. Determination of agerelated increases in large artery stiffness by digital pulse contour analysis. Clin Sci. (2002) 103:371-7. doi: 10.1042/cs1030371

14. Clarenbach CF, Stoewhas AC, van Gestel AJ, Latshang TD, Lo Cascio $\mathrm{CM}$, Bloch KE, et al. Comparison of photoplethysmographic and arterial tonometry-derived indices of arterial stiffness. Hypertens Res. (2012) 35:22833. doi: 10.1038/hr.2011.168

15. Tan L, Latshang TD, Aeschbacher SS, Huber F, Flueck D, Lichtblau M, et al. Effect of nocturnal oxygen therapy on nocturnal hypoxemia and sleep apnea among patients with chronic obstructive pulmonary disease traveling to 2048 meters: a randomized clinical trial. JAMA Network Open. (2020) 3:e207940-e. doi: 10.1001/jamanetworkopen.2020.7940

16. Latshang TD, Lo Cascio CM, Stowhas AC, Grimm M, Stadelmann K, Tesler $\mathrm{N}$, et al. Are nocturnal breathing, sleep, and cognitive performance impaired at moderate altitude (1,630-2,590 m)? Sleep. (2013) 36:1969-76. doi: $10.5665 /$ sleep. 3242

17. Yap YG, Camm AJ. Drug induced QT prolongation and torsades de pointes. Heart. (2003) 89:1363-72. doi: 10.1136/heart.89.11.1363

18. Straus SM, Kors JA, De Bruin ML, van der Hooft CS, Hofman A, Heeringa J, et al. Prolonged QTc interval and risk of sudden cardiac death in a population of older adults. J Am Coll Cardiol. (2006) 47:362-7. doi: 10.1016/j.jacc.2005.08.067

19. Rautaharju PM, Surawicz B, Gettes LS, Bailey JJ, Childers R, Deal BJ, et al. AHA/ACCF/HRS recommendations for the standardization and interpretation of the electrocardiogram: part IV: the ST segment, T and $\mathrm{U}$ waves, and the QT interval: a scientific statement from the American heart association electrocardiography and arrhythmias committee, council on clinical cardiology; the American college of cardiology foundation; and the heart rhythm society. endorsed by the international society for computerized electrocardiology. J Am Coll Cardiol. (2009) 53:982-91. doi: 10.1161/CIRCULATIONAHA.108.191096

20. Akcay M. The effect of moderate altitude on Tp-e interval, Tp-e/QT, QT, cQT and P-wave dispersion. J Electrocardiol. (2018) 51:929-33. doi: 10.1016/j.jelectrocard.2018.07.016

21. Goudis CA, Konstantinidis AK, Ntalas IV, Korantzopoulos P. Electrocardiographic abnormalities and cardiac arrhythmias in chronic obstructive pulmonary disease. Int J Cardiol. (2015) 199:264-73. doi: 10.1016/j.ijcard.2015.06.096

22. Montanez A, Ruskin JN, Hebert PR, Lamas GA, Hennekens CH. Prolonged QTc interval and risks of total and cardiovascular mortality and sudden death in the general population: a review and qualitative overview of the prospective cohort studies. Arch Intern Med. (2004) 164:943-8. doi: 10.1001/archinte.164.9.943

23. Warnier MJ, Rutten FH, Numans ME, Kors JA, Tan HL, de Boer A, et al. Electrocardiographic characteristics of patients with chronic obstructive pulmonary disease. COPD. (2013) 10:62-71. doi: 10.3109/15412555.2012.727918

24. Zupanic E, Zivanovic I, Kalisnik JM, Avbelj V, Lainscak M. The effect of 4-week rehabilitation on heart rate variability and QTc interval in patients with chronic obstructive pulmonary disease. Copd. (2014) 11:659-69. doi: 10.3109/15412555.2014.898046

25. Zulli R, Donati P, Nicosia F, De Vecchi M, Tantucci C, Romanelli G, et al. Increased QT dispersion: a negative prognostic finding in chronic obstructive pulmonary disease. Intern Emerg Med. (2006) 1:279. doi: 10.1007/BF02934761

26. Tirlapur VG, Mir MA. Nocturnal hypoxemia and associated electrocardiographic changes in patients with chronic obstructive airways disease. N Engl J Med. (1982) 306:125-30. doi: 10.1056/NEJM198201213060301
27. Sarubbi B, Esposito V, Ducceschi V, Meoli I, Grella E, Santangelo L, et al. Effect of blood gas derangement on QTc dispersion in severe chronic obstructive pulmonary disease: evidence of an electropathy? Int J Cardiol. (1997) 58:28792. doi: 10.1016/S0167-5273(96)02876-8

28. Tural Onur S, Emet S, Sokucu SN, Onur I. T wave peak-to-end interval in COPD. Int J Chronic Obstr. (2018) 13:2157-62. doi: 10.2147/COPD.S132538

29. Tse G, Gong M, Wong WT, Georgopoulos S, Letsas KP, Vassiliou VS, et al. The Tpeak - Tend interval as an electrocardiographic risk marker of arrhythmic and mortality outcomes: a systematic review and meta-analysis. Heart Rhythm. (2017) 14:1131-7. doi: 10.1016/j.hrthm.2017. 05.031

30. Curkendall SM, DeLuise C, Jones JK, Lanes S, Stang MR, Goehring E, Jr, et al. Cardiovascular disease in patients with chronic obstructive pulmonary disease, Saskatchewan Canada cardiovascular disease in COPD patients. Ann Epidemiol. (2006) 16:63-70. doi: 10.1016/j.annepidem.2005. 04.008

31. Flick MR, Block AJ. Nocturnal vs diurnal cardiac arrhythmias in patients with chronic obstructive pulmonary disease. Chest. (1979) 75:8-11. doi: $10.1378 /$ chest.75.1.8

32. Shepard JW, Jr., Garrison MW, Grither DA, Evans R, Schweitzer PK. Relationship of ventricular ectopy to nocturnal oxygen desaturation in patients with chronic obstructive pulmonary disease. Am J Med. (1985) 78:28-34. doi: 10.1016/0002-9343(85)90457-7

33. Graham WG, Houston CS. Short-term adaptation to moderate altitude. Patients with chronic obstructive pulmonary disease. JAMA. (1978) 240:14914. doi: 10.1001/jama.240.14.1491

34. Edvardsen A, Ryg M, Akero A, Christensen CC, Skjonsberg OH. COPD and air travel: does hypoxia-altitude simulation testing predict in-flight respiratory symptoms? Eur Respir J. (2013) 42:1216-23. doi: 10.1183/09031936.001 57112

35. Akero A, Christensen CC, Edvardsen A, Ryg M, Skjonsberg OH. Pulse oximetry in the preflight evaluation of patients with chronic obstructive pulmonary disease. Aviat Space Environ Med. (2008) 79:518-24. doi: 10.3357/ASEM.2120.2008

36. Gong H, Jr, Tashkin DP, Lee EY, Simmons MS. Hypoxia-altitude simulation test. Evaluation of patients with chronic airway obstruction. Am Rev Respir Dis. (1984) 130:980-6.

37. Eryol NK, Colak R, Ozdogru I, Tanriverdi F, Unal S, Topsakal R, et al. Effects of calcium treatment on QT interval and QT dispersion in hypocalcemia. Am J Cardiol. (2003) 91:750-2. doi: 10.1016/S0002-9149(02) 03423-9

38. Kallergis EM, Goudis CA, Simantirakis EN, Kochiadakis GE, Vardas PE. Mechanisms, risk factors, and management of acquired long QT syndrome: a comprehensive review. Sci World J. (2012) 2012:212178. doi: $10.1100 / 2012 / 212178$

Conflict of Interest: The authors declare that the research was conducted in the absence of any commercial or financial relationships that could be construed as a potential conflict of interest.

Copyright (C) 2021 Bisang, Latshang, Aeschbacher, Huber, Flueck, Lichtblau, Ulrich, Hasler, Scheiwiller, Ulrich, Bloch and Furian. This is an open-access article distributed under the terms of the Creative Commons Attribution License (CC BY). The use, distribution or reproduction in other forums is permitted, provided the original author(s) and the copyright owner(s) are credited and that the original publication in this journal is cited, in accordance with accepted academic practice. No use, distribution or reproduction is permitted which does not comply with these terms. 